\title{
On the blue emission of a novel solution-processed stilbenoid dendrimer thin film for OLED displays
}

\author{
C. Coya ${ }^{\mathrm{a}, *}$, A. de Andrés ${ }^{\mathrm{b}}$, R. Gómez ${ }^{\mathrm{c}}$, C. Seoane ${ }^{\mathrm{c}}$, J.L. Segura ${ }^{\mathrm{c}}$ \\ ${ }^{a}$ Escuela Superior de Ciencias Experimentales y Tecnología, Universidad Rey Juan Carlos, 28933 Madrid, Spain \\ ${ }^{\mathrm{b}}$ Instituto de Ciencia de Materiales de Madrid, CSIC, Cantoblanco, 28049 Madrid, Spain \\ ${ }^{\mathrm{c}}$ Departamento de Química Orgánica, Facultad de Ciencias Químicas, Universidad Complutense, 28040 Madrid, Spain
}

Available online 23 December 2007

\begin{abstract}
A novel blue fluorescent first generation stilbenoid dendrimer built on the 1,3,5-benzene core and endowed with a periphery of hexyloxy branches has been synthesized and is proposed as an active layer for organic light-emitting diodes processed by spin coating. In this work, we have obtained homogeneous thin films with blue emission $(2.8 \mathrm{eV})$ by spin coating instead of the usual evaporation method used for low-weight molecular materials. The absorption and emission spectra of the films are analyzed and compared to that of the molecule. The obtained results show the dependence of the absorption and emission properties on the morphology and the dendrimer aggregation in the film. The effect of inter-dendrimer interactions leads to a broadening of the absorption bands and to a reduction of the threshold as the aggregation increases. The most efficient solid-state emission is for the films obtained from chloroform as solvent in the precursor solution.
\end{abstract}

(C) 2007 Elsevier B.V. All rights reserved.

Keywords: Dendrimer; Photoluminescence; OLED

\section{Introduction}

Since the discovery of efficient electroluminescence (EL) in small organic molecules [1] and conjugated polymers [2] there has been a considerable interest in synthesizing new materials to increase efficiency and stability of organic lightemitting diodes (OLEDs). Linear-chain polymers are the systems most often prepared, but such materials suffer from some limitations such as broad molecular weight distribution, poorly defined morphologies, and uncontrolled intraand inter-chain interactions. Conjugated dendritic structures, however, provide a high degree of control in terms of molecular size, shape, and location of functional groups $[3,4]$. Recently, dendrimers have been shown to be suitable materials for optoelectronic applications [5]. In spite of these advantages, thin films of these materials are usually prepared by evaporation due to the strong tendency to aggregate that prevents their use in low-cost, large-area OLED display technologies, in contrast with polymeric

0022-2313/\$ - see front matter (C) 2007 Elsevier B.V. All rights reserved. doi:10.1016/j.jlumin.2007.12.038 material that are deposited simply by spin coating or even printing [6]. Previous attempts to obtain thin films with blue emission from solution-processed dendrimers based on stilbene report a broad, yellow emission due to the strong aggregation [7]. In this work, a new blue-emitting stilbenoid dendrimer has been synthesized and thin films of this material have been prepared by spin coating. Photoluminescence (PL) recorded from these films shows a well-resolved blue band with maximum intensity at $2.83 \mathrm{eV}$, making them suitable candidates as active layer for blue OLED displays.

\section{Experimental section}

\subsection{Optical measurements}

Absorption and emission spectra have been measured both from solutions in $\mathrm{CHCl}_{3}$ and films on quartz substrates. Absorption spectra were recorded with a Varian spectrophotometer model Cary 4000. PL measurements were performed at room temperature under $365 \mathrm{~nm}$ excitation from a quartz tungsten lamp with a $10 \mathrm{~nm}$ bandpass filter. A home-made system consisting of a Jobin-Yvon HR

\footnotetext{
${ }^{*}$ Corresponding author.

E-mail address: carmen.coya@urjc.es (C. Coya).
} 
460 monochromator coupled to a $\mathrm{N}_{2}$-cooled $\mathrm{CCD}$ was used. The light was collected through an Olympus microscope. All emission spectra have been corrected by the spectral response of the experimental setup.

\subsection{Synthesis}

The novel dendrimer (compound 3 in Scheme 1) was obtained with $30 \%$ yield following the Wittig-Horner reaction between triphosphonate (2) and p-hexyloxybenzaldehyde (1) in dry tetrahydrofuran (THF), using potassium tert-butoxide as base. This synthetic approach is highly convenient, as it provides stilbenoid dendrimers straightforwardly and in good yields. Moreover, all-trans configuration for the double bonds is attained as an additional advantage of this approach, thus avoiding the need of further isomerization steps. This all-trans configuration is evidenced by ${ }^{1} \mathrm{H}-\mathrm{NMR}$. In fact, besides the expected signals for both the benzene core (a singlet at $7.84 \mathrm{ppm}$ ) and the peripheral p-alkoxybenzene rings (two doublets with a characteristic ortho coupling constant $J$ of ca. $8.68 \mathrm{~Hz}$ ), two additional doublets, with a typical coupling of ca. $16.34 \mathrm{~Hz}$, are also observed. This value corresponds well with the expected value for a ${ }^{3} J_{\text {trans }}$ coupling, thus evidencing the trans disposition of the vinylic protons [8]. Further evidence concerning the structure of 3 is given by ${ }^{13} \mathrm{C}-\mathrm{NMR}$, FTIR spectroscopies, and mass spectrometry (MS). The good accordance between calculated and expected values for microanalysis gives a proof of the purity of the material.

\subsection{Film fabrication}

All the solutions were kept in an ultrasonic bath for $10 \mathrm{~min}$ and filtered through a $0.2 \mu \mathrm{m}$ filter, prior to spin

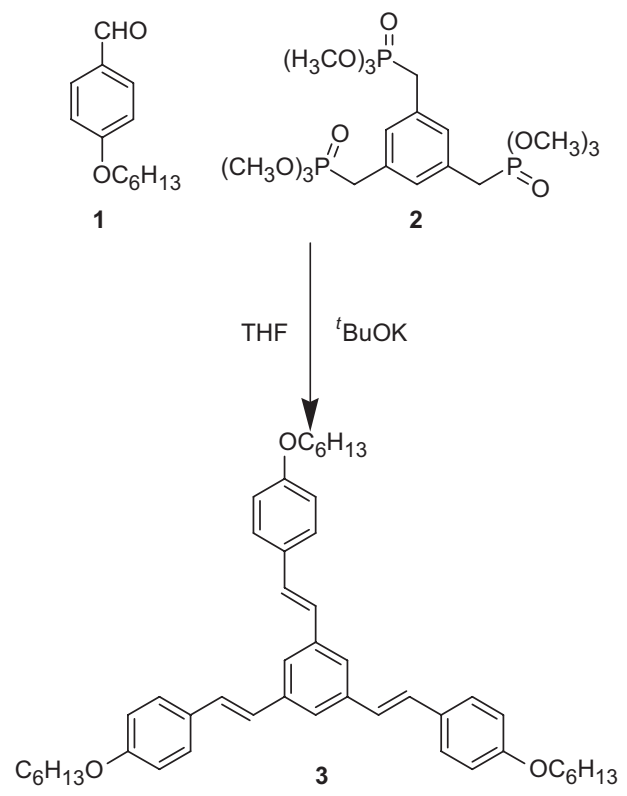

coating at $6000 \mathrm{rpm}$ for $45 \mathrm{~s}$ on quartz substrates and then cured for $30 \mathrm{~min}$ at $60^{\circ} \mathrm{C}$. All the processes were carried out in a clean room environment class 1000 . The concentration was for all cases $0.9 \mathrm{wt} \%$.

\section{Results and discussion}

The interesting photophysical and photochemical properties of stilbenoid derivatives have motivated the incorporation of stilbenoid chromophores into dendrimers. Only recently, several synthetic routes have been developed to achieve this target. The introduction of different chromophores, both at peripheral positions and at the core, arises as a useful approach for the appropriate tuning of properties. The introduction of peripheral alkoxy chains enhances the solubility in usual organic solvents and increases the electronic density of the molecule since oxygen is an electronic donor. We have studied the effect of these peripheral alkoxy chains on the final material comparing the dendrimer 3 with its unsubstituted analogue: 1,3,5-tristyrylbenzene (4, Fig. 1). The absorption and emission spectra of both compounds in $10^{-5} \mathrm{M}$ chloroform $\left(\mathrm{CHCl}_{3}\right)$ solutions are shown in Figs. 1(a) and (b) respectively. A red shift is observed both on absorption and emission spectra as expected due to the addition of an electron-donating group [9]. The maximum of the absorption is at $3.82 \mathrm{eV}$ for dendrimer 3 and at $3.93 \mathrm{eV}$ for the unsubstituted analog 4 . The narrower absorption band for the dendrimer indicates that no aggregation occurs, confirming the increase of solubility. A large increase in the molar absortivity coefficient $\varepsilon$ for the dendrimer is observed $\left(66900 \mathrm{M}^{-1} \mathrm{~mm}^{-1}\right.$ for 3 and $5700 \mathrm{M}^{-1} \mathrm{~mm}^{-1}$ for 4 at the maximum of their absorption spectra). The dendrimer emission peaks at $3.09 \mathrm{eV}(0.1 \mathrm{eV}$ red shifted with respect to its analog), with a vibronic peak at $2.98 \mathrm{eV}$, and a shoulder at $2.83 \mathrm{eV}$.

Obtaining uniform thin films is the first technical requisite to fabricate an OLED display. For this purpose tests with four different solvents have been made in order to obtain suitable morphologies: toluene, THF, chlorobenzene $\left(\mathrm{C}_{6} \mathrm{H}_{5} \mathrm{Cl}\right)$, and $\mathrm{CHCl}_{3}$. Although all the dendrimer solutions are transparent at first glance, this does not guaranty homogeneous films after the deposition due to the tendency to form dendrimer islands of these low-weight molecular materials. We analyzed the absorption and emission of the thin films fabricated by spin coating and compared them to the molecule spectra obtained from a $10^{-5} \mathrm{M}$ solution in $\mathrm{CHCl}_{3}$.

Environmental scanning electron microscopy (ESEM) images (Fig. 2) reveal that the final morphology results of the films to be very different depending on the solvent. We could observe a relation between the morphology of the films and their absorption spectra. The spectra of films obtained from toluene could not be measured due to the formation of highly non-homogeneous films with dendrimer islands and large areas with no material (white zones in 


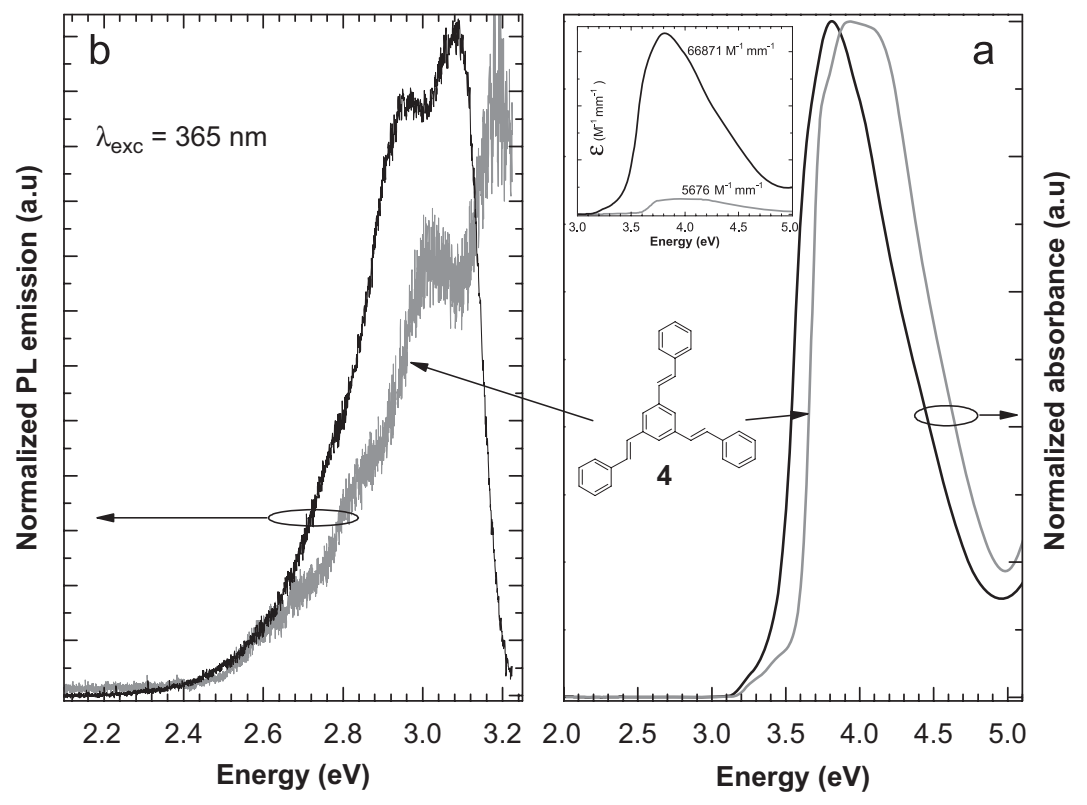

Fig. 1. Comparison of the absorption (a) and emission (b) spectra of the unsubstituted $1,3,5$-tristirylbenzene core (4) and the dendrimer in $10^{-5} \mathrm{M}$ chloroform solution. The inset shows the values of the molar extinction coefficient $\varepsilon\left(\mathrm{M}^{-1} \mathrm{~mm}^{-1}\right)$.
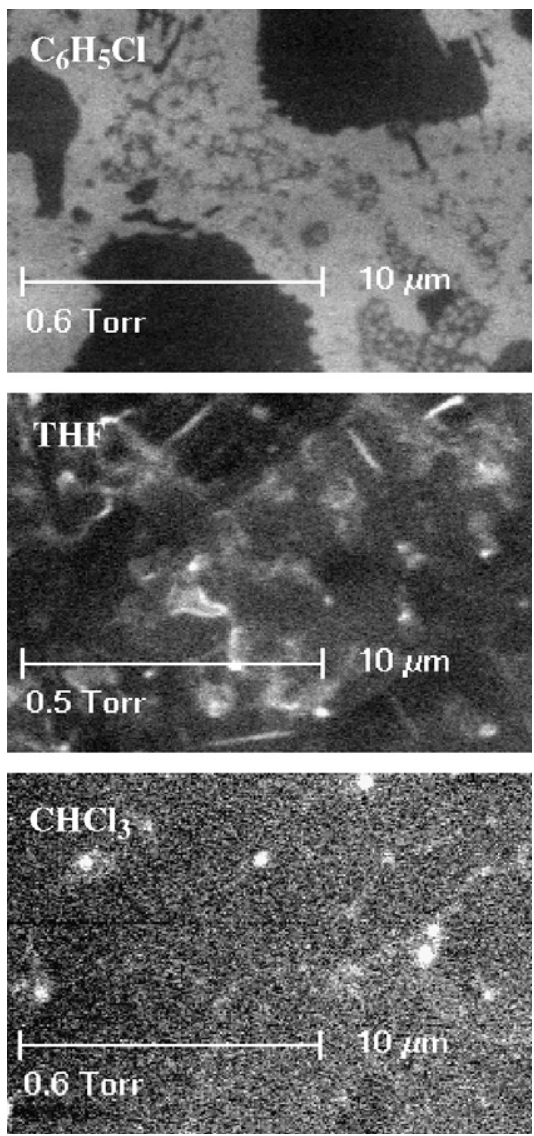

Fig. 2. ESEM images of the obtained films.

Fig. 2), as confirmed by energy dispersion X-ray spectrometry (EDX).

The absorption and emission spectra of the other films are shown in Fig. 3, together with the spectra for the

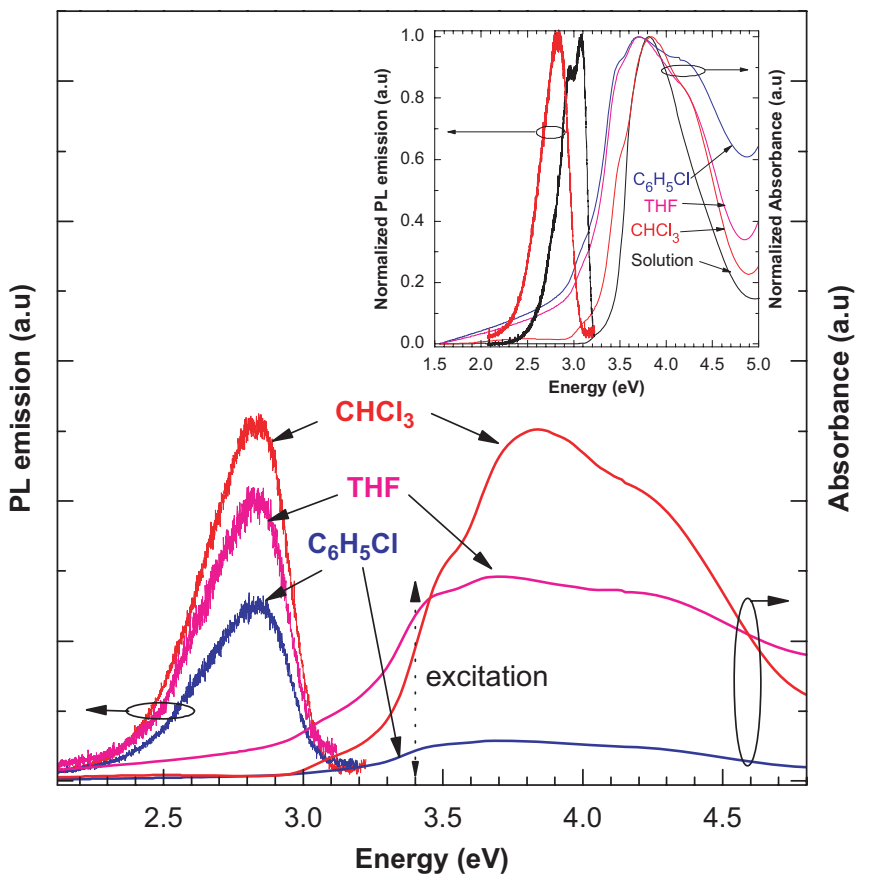

molecule in $\mathrm{CHCl}_{3}$ solution, shown for comparison. The absorption spectra of the films from $\mathrm{C}_{6} \mathrm{H}_{5} \mathrm{Cl}$ and THF solvents show a shift to the red with respect to the material in $\mathrm{CHCl}_{3}$ solution (inset of Fig. 3), a broadening of the absorption bands, and the appearance of new bands revealing the effect of inter-dendrimer interaction, which lead to the reduction of the absorption threshold energy. 
The broader spectrum corresponds to the films where larger dendrimer aggregate areas are observed. On the contrary, the film obtained from $\mathrm{CHCl}_{3}$ as the precursor solvent does not show a significant shift in the maximum of absorption and exhibits a narrower spectrum. The film is transparent with a very good uniformity. The measured thickness of this film is $185 \mathrm{~nm}$ and the roughness is around $\pm 5 \mathrm{~nm}$. The emission for all the fabricated films peaks at $2.83 \mathrm{eV}$ and lacks vibrational structure (Fig. 3). The red shift, observed in the solid-state PL emission when compared with the molecule, may be explained by the fact that in the solid state the molecules can experience a wider distribution of conformations, including a more planar state that gives rise to a lower HOMO-LUMO energy gap [10].

Fig. 3 also shows the emission and absorption spectra of the films in a suitable scale in order to compare their relative intensities. Although the high absorbance at the excitation wavelength is for the film obtained with THF as solvent in the precursor solution, the maximum emission corresponds to the most homogeneous film obtained with chloroform as solvent in the precursor solution.

\section{Conclusions}

The attachment of alkoxy $\left(-\mathrm{OC}_{6} \mathrm{H}_{13}\right)$ chains to a $1,3,5-$ tristyrylbenzene core results in a blue-emitting stilbenoid dendrimer both in solution and in thin solid film. We have fabricated uniform blue-emitting thin films by spin coating using chloroform as solvent in the precursor solution, which are suitable candidates to fabricate OLED displays with low-cost, large-area solution-processing technologies.

\section{Acknowledgments}

Financial support for this work was by Comunidad Autónoma de Madrid, Universidad Rey Juan Carlos under Project URJC-TIC-044-1 (2005-2007) and by the Ministerio de Educación y Ciencia (Spain) under Projects CTQ2004-03760 and MAT2006-01004 and Ramón y Cajal program (R. Gómez). The authors are grateful to Dr. Angel Luis Alvarez for the thickness measurements.

\section{Reference}

[1] [a] J. Dresner, RCA Rev. 30 (1969) 322; [b] W. Helfrich, W.G. Scheider, Phys. Rev. Lett. 14 (1965) 229.

[2] A. Kraft, A.C. Grimsdale, A.B. Holmes, Angew. Chem. Int. Ed. 37 (1998) 402.

[3] S.M. Grayson, J.M.J. Fréchet, Chem. Rev. 101 (2001) 3819.

[4] E. Díez-Barra, J.C. García-Martinez, R. del Rey, J. RodríguezLópez, F. Giacalone, J.L. Segura, N. Martín, J. Org. Chem. 68 (2003) 3178.

[5] [a] C.S. Cameron, C.B. Gorman, Adv. Funct. Mater. 12 (2002) 17.

[6] T.R. Hebner, C.C. WU, K. Marcy, M.H. Lu, J.C. Strum, Appl. Phys. Lett. $72(1998) 519$.

[7] T.W. Kwon, M.M. Alam, S.A. Jejekhe, Chem. Mater. 16 (2004) 4657.

[8] D.M. Guldi, C. Luo, A. Swartz, R. Gómez, J.L. Segura, N. Martín, J. Org. Chem. 67 (2002) 1141.

[9] M.L. Renak, G.P. Bartholomew, S. Wang, P.J. Ricato, R.J. Lachicotte, G.C. Bazan, J. Am. Chem. Soc. 121 (1999) 7787.

[10] M. Halim, J.N. G Pillow, I.D.W. Samuel, P.L. Burn, Adv. Mater. 11 (1999) 371 